\title{
Estudio comparativo entre la difusión de U en Zry-4 y en Zr-a puro
}

\section{Comparative study of $U$ diffusion in
Zry-4 and pure $\alpha-Z r$}

Rodolfo Ariel Pérez ${ }^{12,3,}$ Jorge Apóstol Gordillo ${ }^{1}$, Manuel Iribarren ${ }^{1,2}$, Nicolás Di Lalla ${ }^{3}$

\footnotetext{
${ }^{1}$ Gerencia de Materiales, Comisión Nacional de Energía Atómica (CNEA), Avenida General Paz 1499, (B1650KNA) San Martín, Buenos Aires, Argentina.

e-mail: rodperez@cnea.gov.ar

${ }^{2}$ Instituto Sabato - UNSAM/CNEA, Av. G. Paz 1499 (B1650KNA), San Martín, Buenos Aires, Argentina.

${ }^{3}$ Consejo Nacional de Investigaciones Científicas y Técnicas- CONICET, Buenos Aires, Buenos Aires, Argentina.
}

\begin{abstract}
RESUMEN
Por medio de la espectrometría $\alpha$ se estudió la difusión de U en Zry-4 y se la comparó con la difusión en Zr- $\alpha$ puro en el rango de temperaturas que va de los 570 a los $820^{\circ} \mathrm{C}$ a fin de analizar el proceso difusivo en ambas matrices, sus similitudes y sus diferencias. Los valores del coeficiente de difusión $D$ son sistemáticamente mayores en Zry-4, en tanto que su dependencia con la temperatura obedece a la ley de Arrhenius siendo $D_{0}$ $=(3,2 \pm 1,1) \mathrm{m}^{2} / \mathrm{s}$ y $Q=(316 \pm 9) \mathrm{kJ} \mathrm{mol}^{-1}$ en contraposición con el $\mathrm{Zr}-\alpha$ que presenta un apartamiento a dicha ley consistente en una curvatura en el gráfico ln $(D)$ vs $1 / \mathrm{T}$. Por debajo de $600{ }^{\circ} \mathrm{C}$ la solubilidad del U en Zry-4 es menor que en $\mathrm{Zr}$ puro.
\end{abstract}

Palabras clave: Difusión, espectrometría- $\alpha$, Uranio, Zirconio, Zircaloy.

\section{ABSTRACT}

Throughout the $\alpha$-spectrometry $U$ diffusion in pure Zry-4 was studied and compared with pure $\alpha-\mathrm{Zr}$ in the temperature range 843-1093 $\mathrm{K}$ in order to analyze the diffusion process in both matrices. $D$ values are systematically higher in Zry-4 and its temperature dependence obeys the Arrhenius law being: $D_{0}=(3,2 \pm 1,1)$ $\mathrm{m}^{2} / \mathrm{s}$ and $Q=(316 \pm 9) \mathrm{kJ} \mathrm{mol}^{-1}$, whereas in $\alpha-\mathrm{Zr} \mathrm{U}$ diffusion present an apart to this law, consistent in a downward curvature in the $\ln (D)$ vs $1 / \mathrm{T}$ plot. Below $873 \mathrm{~K} \mathrm{U}$ solubility in Zry4 is lower than in pure $\mathrm{Zr}$.

Keywords: Diffusion, $\alpha$-spectrometry, Uranium, Zirconium, Zircaloy.

\section{INTRODUCCIÓN}

Recientemente en nuestro laboratorio hemos implementado la aplicación de la espectrometría $\alpha$ a la determinación de perfiles de U en metales de uso en la industria nuclear [1]. Dada la posibilidad de determinar perfiles submicrométricos con esta técnica, ahora es posible medir coeficientes de difusión $(D)$ de U a temperaturas menores a las reportadas en la literatura [2]; la influencia de la difusión por caminos rápidos puede hacerse despreciable en las nuevas mediciones si el tamaño de grano de las muestras es pequeño comparado con el camino libre medio de difusión estudiado.

Los datos existentes sobre la difusión de $\mathrm{U}$ en metales puros que se encuentran en la literatura [2] son escasos, mayormente medidos en las décadas del 60 y 70, en materiales pobremente caracterizados y a temperaturas muy por encima a las de uso en reactores nucleares. Para ello se utilizaron técnicas como la autorradiografía, la radiografía de fragmentos de fisión y la actividad residual, todas las cuales requieren analizar perfiles de, como mínimo, varias decenas de micrones de profundidad.

Al aplicar la espectrometría $\alpha$ para medir la difusión de U en Ti- $\alpha$ [3] se pudo extender el rango de temperatura de estudio desde aproximadamente la de transición $\mathrm{T}_{\alpha / \beta}$ hasta $540{ }^{\circ} \mathrm{C}$, encontrándose una dependencia con la temperatura de $D$ que sigue la ley de Arrhenius: $D(T)=D_{0} \exp (-Q / R T)$, siendo R la constante de los gases ideales, obteniendo valores de la energía de activación $Q$ y del factor pre-exponencial $D_{0}$ próximos a los valores de auto-difusión en Ti- $\alpha$ [4], consistente con la difusión de una impureza vía un mecanismo de vacancias. La única medición previa hecha por FEDOROV et al [5] en un rango de temperaturas más cor- 
to $\left(851\right.$ a $\left.747^{\circ} \mathrm{C}\right)$ muestra una energía de activación no sólo menor al $Q$ de auto-difusión, sino a la energía de formación de vacancias en el Ti- $\alpha$, todo lo cual indica que los valores así medidos no se corresponden con $D$ a dilución infinita sino con coeficientes de difusión efectivos en donde los valores de $D$ en volumen están afectados por la presencia de caminos rápidos.

Ante esta evidencia experimental concluimos que era necesario medir en algunos casos y remedir en otros la difusión de U en materiales de uso en la industria nuclear usando la espectrometría $\alpha$. En el caso particular de $\mathrm{Zr}$, sólo había mediciones en la fase $\beta$, bcc, [6], hasta que recientemente en nuestro laboratorio medimos la difusión de $\mathrm{U}$ en $\mathrm{Zr}$ en su fase $\alpha$, hep, [7] en un rango extendido de temperaturas $\left(540\right.$ a $\left.850{ }^{\circ} \mathrm{C}\right)$ obteniendo valores muy próximos a los de la autodifusión en $\mathrm{Zr}-\alpha$. También se observó que la dependencia de $D$ del U con la temperatura no obedece la ley de Arrhenius, mostrando una curvatura en el gráfico $\ln (D)$ vs $1 / T$ similar a la medida en el caso de la autodifusión en $\mathrm{Zr}-\alpha$.

En el presente trabajo medimos en forma simultánea la difusión de U en pares de muestras de $\mathrm{Zr}$ puro y Zircaloy 4 (Zry-4) a fin de analizar el efecto sobre el proceso difusivo de los distintos aleantes presentes. El Zry-4 es una marca comercial que se le da a una aleación base $\mathrm{Zr}$ usada como vaina combustible en reactores nucleares. Los principales aleantes del Zry-4 usado en este trabajo son: $\mathrm{Cr}$ : 0.065; Sn: 1.5 ; Fe: 0.21 y Ni: 0.0032, donde todos los valores están dados en porcentaje en peso.

\section{MATERIALES Y MÉTODOS}

\subsection{Caracterización de muestras y preparación de los pares difusivos}

Las mediciones se realizaron sobre muestras de Zry-4 consistentes en discos de $12 \mathrm{~mm}$ de diámetro y de entre 5 y 6 mm de espesor, y sobre las mismas muestras de $\mathrm{Zr}$ de $99.97 \%$ de pureza que fueran utilizadas en el trabajo [7].

Dichos materiales fueron pulidos primero mecánicamente con papeles abrasivos hasta granulometría 1000 y luego con un pulido mecánico/químico, en paño de terciopelo sobre el que se deposita bicromato de amonio en cenizas con unas gotas de ácido fluorhídrico al $0.5 \%$ molar. Se obtiene así una superficie espejada de una rugosidad inferior a los $10 \mathrm{~nm}$, verificada por interferometría óptica.

Los pares de difusión fueron construidos por sublimación en vacío de U empobrecido de $99.97 \%$ de pureza, sobre la superficie de las muestras, calentando por efecto Joule un filamento de W. Se trabajó en un vacío del orden de $10^{-6}$ torr, obteniéndose películas delgadas de un espesor de alrededor de $10 \mathrm{~nm}$.

Los recocidos se realizaron en un vacío dinámico de $2 \times 10^{-6}$ torr para tiempos inferiores a $2 \times 10^{5} \mathrm{seg}$. Para tiempos mayores se pusieron los pares difusivos en tubos de cuarzo sellados en atmósfera de Ar de alta pureza. Para prevenir reacciones entre el $\mathrm{Zr}$ y el cuarzo las muestras se envolvieron en papel de Ta.

Se utilizaron hornos eléctricos controlados con termopares tipo S de Pt-PtRh; lo que permite controlar las temperaturas de los tratamientos térmicos con una precisión de $\pm 1 \mathrm{~K}$.

Los recocidos de difusión en la muestras de Zry-4 fueron realizados conjuntamente con las muestras de $\mathrm{Zr}-\alpha$ en varias de las temperaturas estudiadas (aquellas que fueran publicadas en [7]). En estos casos fue posible comparar las variaciones en los valores de sus coeficientes de difusión a una misma temperatura descartando cualquier error sistemático que pudiese ocurrir durante el proceso experimental. En otras, particularmente a la temperatura más baja, que requiere de recocidos más largos, sólo se midió en Zry-4. 


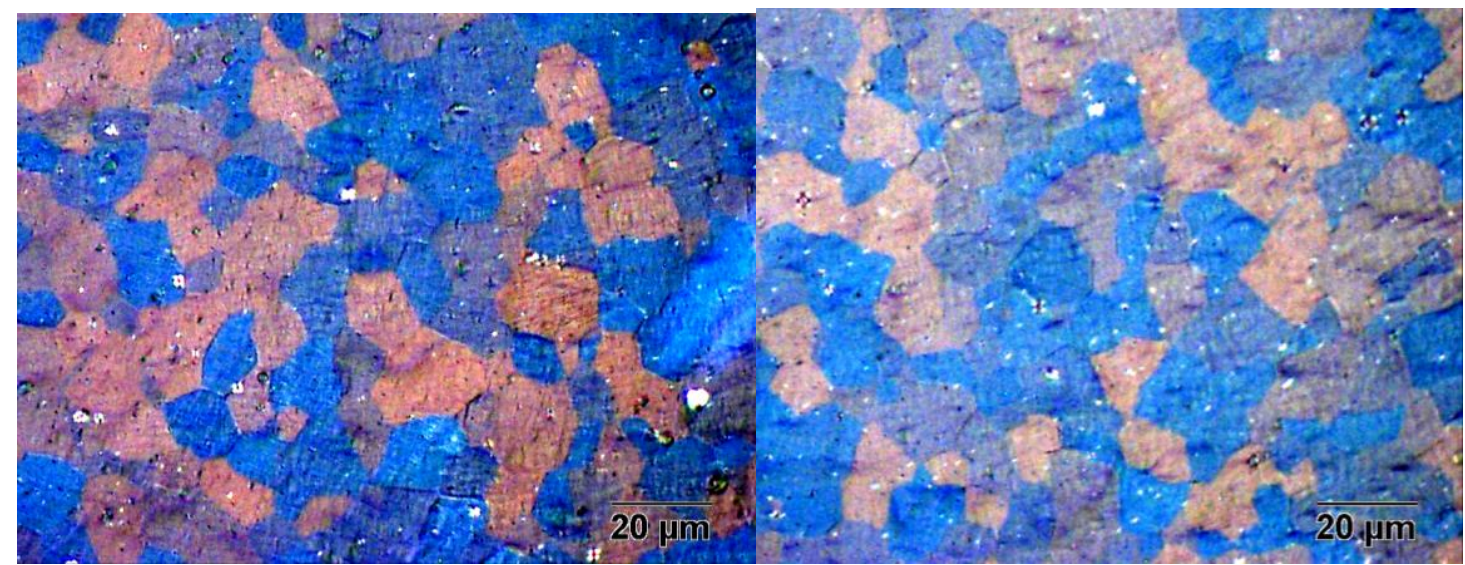

Figura 1: Micrografías de Zry-4: a) La imagen de la derecha corresponde a una muestra que fue tratada térmicamente a $1074 \mathrm{~K}\left(801^{\circ} \mathrm{C}\right) 23 \mathrm{hs}$; b) la imagen de la izquierda corresponde a una probeta sin tratamiento térmico previo.

Se tuvo particular cuidado en no realizar ningún tratamiento previo sobre las muestras de Zry-4 a fin de no alterar sus propiedades, en particular la microestructura de las distintas fases que componen esta aleación.

En las Fig.1 a) y b), se muestran las microestructuras típicas del Zry-4 utilizado antes y después de los recocidos de difusión; no se observa ninguna diferencia importante entre ambas, por lo que podemos concluir que no ocurrieron alteraciones significativas en las muestras durante el proceso difusivo.

\subsection{Descripción de la espectrometría a}

La espectrometría $\alpha$ permite comparar los espectros de las partículas $\alpha$ emitidas por el $U$ antes y después del recocido de difusión, aprovechar el hecho de que las partículas $\alpha$ al interactuar con la materia pierden energía en forma proporcional a la distancia recorrida y de esta forma determinar un perfil de difusión.

La medición de la energía y el número de las partículas $\alpha$ emitidas por el U se hizo con un detector de barrera de silicio modelo Camberra PD 150-16-100-AM, que posee una superficie activa de $150 \mathrm{~mm}^{2}$ y una resolución en energías de aproximadamente $16 \mathrm{keV}$.

El espectro inicial (muestra sin recocido) fue ajustado por una Campana de Gauss cuyo ancho está dado, principalmente, por la convolución del espesor del depósito de U, el ruido electrónico del sistema de medición y la diferencia en la trayectoria de las partículas $\alpha$ dadas por el ángulo sólido subtendido entre los distintos puntos de la superficie de la muestra y la superficie activa del detector. Otras contribuciones al ruido tales como el straggling pueden ser despreciadas, excepto la rugosidad de la superficie de la muestra, si estas no están lo suficientemente bien pulidas. En nuestro caso particular, el ancho inicial de la Campana de Gauss fue de $70 \mathrm{keV}$.

En [1] hay una descripción completa de la aplicación de la espectrometría $\alpha$ a la medición de perfiles de difusión en sólidos en las condiciones existentes en nuestro laboratorio.

La calibración en energías (conversión de número de canal a energía, keV) se realizó utilizando una fuente triple de calibración patrón de ${ }^{241} \mathrm{Am},{ }^{239} \mathrm{Pu} \mathrm{y}{ }^{244} \mathrm{Cm}$, una fuente de y ${ }^{233} \mathrm{U}$ y los propios picos de los espectros $\alpha$ del U, correspondientes al ${ }^{238} \mathrm{U}$ de $4267 \mathrm{keV}$ y al ${ }^{235} \mathrm{U}$ de $4679 \mathrm{keV}$. 


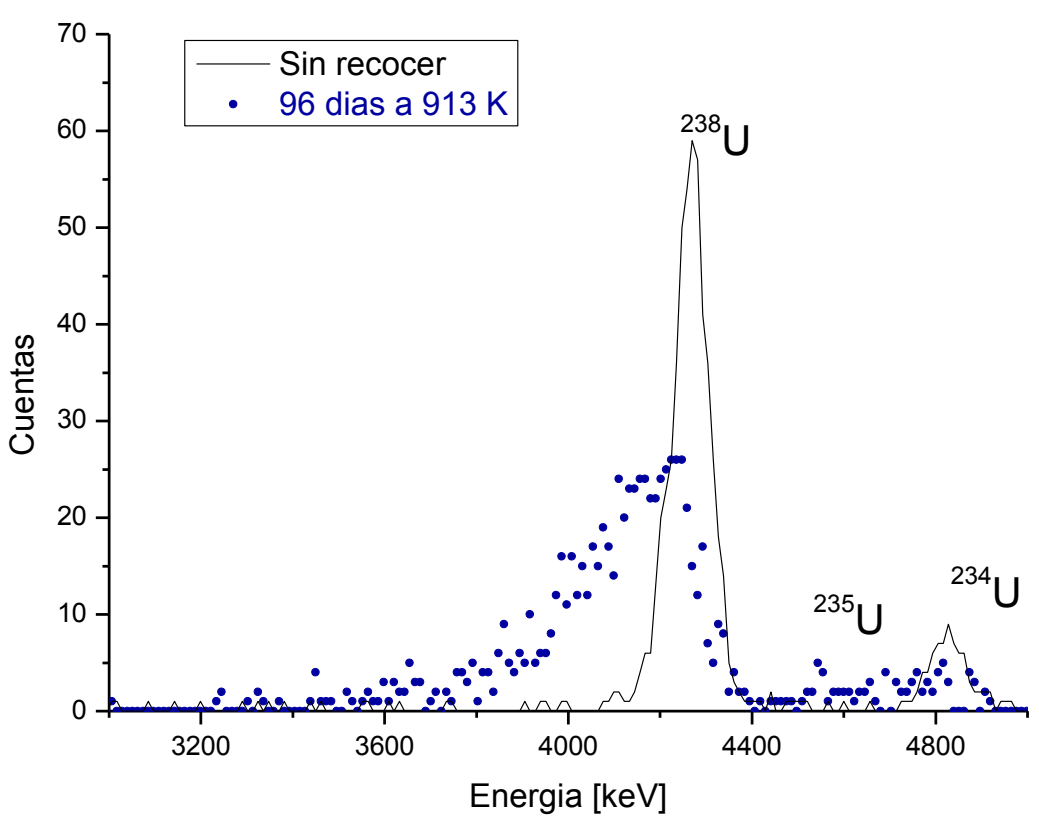

Figura 2: Espectros $\alpha$ antes (línea llena) y después (puntos) de hacer un recocido a $640{ }^{\circ} \mathrm{C}$ durante 96 días.

En la Figura 2, en línea llena, se muestra un espectro proveniente de la emisión de partículas $\alpha$ obtenido después de depositar el U sobre una muestra típica, antes de hacer el recocido de difusión (centraremos todo el análisis en las emisiones del isótopo mayoritario ${ }^{238} \mathrm{U}$ de $4267 \mathrm{keV}$ ).

Luego de efectuado el recocido se toma un nuevo espectro (puntos en la Figura 2) que muestra un ensanchamiento respecto del previo, producto de la pérdida de energía de las partículas $\alpha$ que son emitidas, no ya desde la superficie, sino desde distintas profundidades, de acuerdo a la redistribución del U por efecto del proceso difusivo; a mayor profundidad corresponde una mayor pérdida de energía por interacción con los iones de la matriz.

El valor de esta pérdida viene dado por el poder de frenado, $\mathrm{dE} / \mathrm{dx}$ (en inglés stopping power) de la partícula $\alpha$ en la materia que está definido, por ejemplo, en la ref. [8] y que puede ser calculado por la subrutina "stopping range" del programa SRIM 2008 [9] para casi cualquier elemento de la tabla periódica y para sus aleaciones. Para energías mayores a $1 \mathrm{MeV}$, que son a las que estaremos trabajando, el frenado ocurre mayoritariamente por la interacción electrónica y puede ser ajustado por una función parabólica:

$$
d E / d x(E)=a+b \cdot E+c \cdot E^{2}
$$

donde E es la energía de la partícula medida en $\mathrm{keV}$ y, aplicado al Zry-4: a = 669,913848 eV nm ${ }^{-1}, \mathrm{~b}=$ $-1,5731277 \times 10^{-4} \mathrm{~nm}^{-1}$ y c $\left.=1,4080616 \times 10^{-11}\right) \mathrm{eV}^{-1} \mathrm{~nm}^{-1}$. A partir de ello, cuando una partícula $\alpha$ es emitida por un átomo de $\mathrm{U}$ que está a una profundidad $\mathrm{x}$, ésta puede calcularse a través de la expresión:

$$
x=\int_{E_{0}}^{E_{d}} \frac{d E}{d E / d x}=\int_{E_{0}}^{E_{d}} \frac{d E}{a+b E+c E^{2}}
$$

donde $E_{0}=4267 \mathrm{keV}$ es la energía de emisión de la partícula $\alpha$ (para ${ }^{238} \mathrm{U}$ ) mientras que $E_{d}$ es la energía con la que es detectada cuando llega a la superficie.

Esta expresión es integrable analíticamente y tiene como solución:

$$
\boldsymbol{x}\left(\boldsymbol{E}_{\boldsymbol{d}}\right)=17552\left[-0,31515-\arctan \left(2,47144 \times 10^{-7} \boldsymbol{E}_{\boldsymbol{d}}-1,38058\right)\right]
$$

En ella, $\boldsymbol{E}_{\boldsymbol{d}}$ debe ser expresada en $\mathrm{eV}$, y x en nm; arctan es la función arco-tangente.

De esta forma es posible obtener un perfil de difusión del tipo Concentración [número de cuentas del espectro para una dada $E_{d}$ ] vs Profundidad [x proveniente de la ec.(3)] 


\section{RESULTADOS}

Los resultados y el análisis que sigue, se basan en la información obtenida a partir de los espectros detectados antes y después del recocido de difusión, Figura 2.

Si la solubilidad sólida del U en la matriz es menor que la cantidad de U ingresado a la matriz a partir del depósito inicial, la solución a la ley de Fick para la difusión con estas condiciones iniciales y de contorno (depósito delgado), nos lleva a una solución de tipo Campana de Gauss:

$$
C(x)=\frac{C_{0}}{\sqrt{\pi D\left(t+t_{0}\right)}} \exp \left(\frac{-x^{2}}{4 D\left(t+t_{0}\right)}\right)
$$

donde $C$ es la concentración de $\mathrm{U}$ a la profundidad $x, C_{0}$ es la cantidad inicial de $\mathrm{U}$ por unidad de superficie, $D$ es el coeficiente de difusión a la temperatura de trabajo, $t$ es el tiempo de recocido y $t_{0}$ es un tiempo definido ad-hoc para poder descontar el perfil inicial y, por ende, el error sistemático asociado al sistema de medición.

La forma más representativa de mostrar los perfiles de difusión medidos para cada temperatura consiste en linealizar la ec.(4), graficar el $\ln \left(C / C_{0}\right)$ vs $x^{2}$ y ajustar estos valores usando cuadrados mínimos; de la pendiente de este ajuste $(p)$ es posible obtener el valor de $D$ para cada temperatura estudiada:

$$
\boldsymbol{D}=\frac{\boldsymbol{p}-\boldsymbol{p}_{0}}{4 \boldsymbol{t} \boldsymbol{p} \boldsymbol{p}_{0}}
$$

siendo $p_{0}$ la pendiente de la recta de ajuste del perfil inicial.

En la Figura 3 se muestran estos gráficos con sus correspondientes ajustes para cada temperatura. En la Tabla 1 se resumen todos los valores de D para el Zry-4 juntamente como las temperaturas y tiempos de recocido, donde el error reportado se obtiene del ajuste de cuadrados mínimos conjuntamente con la variación en el valor de D al modificar la selección de corte de la porción del espectro a analizar, en unos pocos puntos en las bajas energías. Se adjuntan los valores de D obtenidos para la difusión de U en $\mathrm{Zr}-\alpha$ [7] a esas mismas temperaturas.

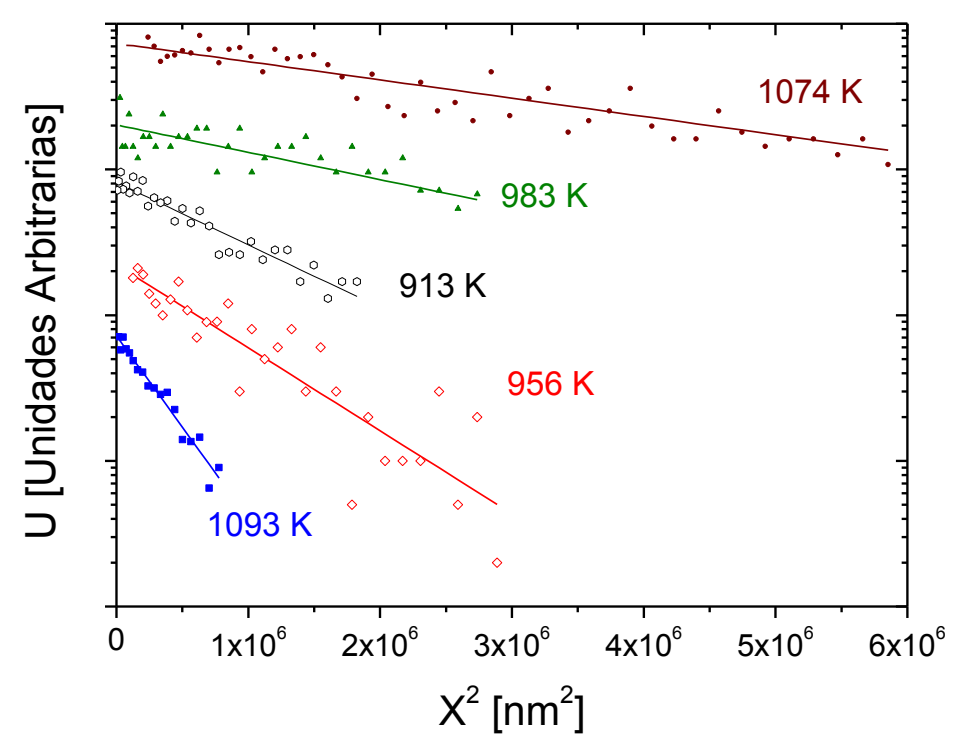

Figura 3: Perfiles de difusión para cada temperatura (ec.(4) linealizada). 
Tabla 1: Valores de los coeficientes de difusión de U medidos en Zry-4 junto con los presentados en [7].

\begin{tabular}{ll|l|l|l}
\hline $\mathbf{T}(\mathbf{K})$ & $\mathbf{(} \mathbf{C})$ & TIEMPO $\left(\mathbf{1 0}^{\mathbf{4}} \mathbf{S}\right)$ & $\mathbf{D}_{\mathrm{ZRY}-4}\left(\mathbf{M}^{2} \mathbf{S}\right)$ & $\mathbf{D}_{\mathrm{ZR}}\left(\mathbf{M}^{\mathbf{2}} \mathbf{S}\right)[\mathbf{7}]$ \\
\hline 1093 & 820 & 0,36 & $(2,2 \pm 0,2) \times 10^{-17}$ & $(6.3 \pm 0.6) * 10^{-18}$ \\
\hline 1074 & 801 & 8,2 & $(1,0 \pm 0,1) \times 10^{-17}$ & $(4.5 \pm 0.5) * 10^{-18}$ \\
\hline 983 & 710 & 10,08 & $(6,5 \pm 1,5) \times 10^{-19}$ & $(2.5 \pm 0.3) * 10^{-19}$ \\
\hline 956 & 683 & 86,4 & $(2,1 \pm 0,4) \times 10^{-19}$ & - \\
\hline 913 & 640 & 397,44 & $(3,0 \pm 0,2) \times 10^{-20}$ & $(4.8 \pm 0.8) * 10^{-20}$ \\
\hline 863 & 590 & 1071,36 & $(2,1 \pm 0,6) \times 10^{-21}$ & $(1,5 \pm 0,3) \times 10^{-21}$ \\
\hline 843 & 570 & 5270 & $(6,1 \pm 2,0) \times 10^{-22}$ & - \\
\hline
\end{tabular}

Todos estos valores son presentados en un gráfico tipo Arrhenius, ln (D) vs 1/T, en la Figura 4, en la que también se grafica la curva de auto-difusión en $\mathrm{Zr}-\alpha$ [10] a fines comparativos.

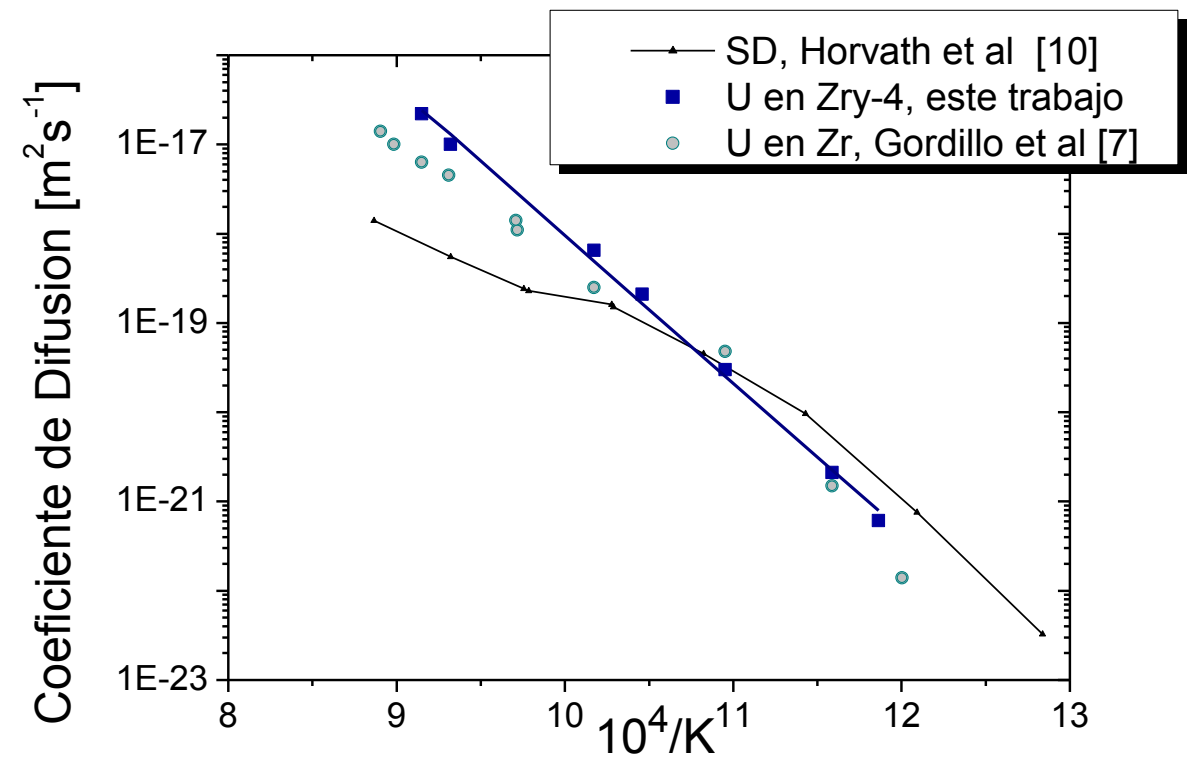

Figura 4: Dependencia de D del U con la temperatura para Zry-4, Zr-a puro y para la auto-difusión en Zr- $\alpha$.

\section{DISCUSIÓN}

Al comparar los datos presentados en el gráfico de Arrhenius de la figura 4, podemos notar dos diferencias importantes: por un lado que los valores del coeficiente de difusión del U a dilución infinita medidos en Zry4 son sistemáticamente mayores a los medidos en $\mathrm{Zr}$ puro a partir de $l o s \quad 600{ }^{\circ} \mathrm{C}$ en adelante $\mathrm{y}$, fundamentalmente, que mientras que los valores medidos en $\mathrm{Zr}$ no cumplen con la ley de Arrhenius, las mediciones en Zry-4 si lo hacen, pudiendo calcularse entonces una entalpía de activación $Q=(316 \pm 9) \mathrm{kJ} / \mathrm{mol}$ y un factor pre-exponencial $\mathrm{D}_{0}=(3,2 \pm 1,1) \times 10^{-2} \mathrm{~m}^{2} / \mathrm{s}$ :

$$
\boldsymbol{D}(\boldsymbol{T})=(3,2 \pm 1,1) \times 10^{-2} \exp \left(-\frac{(316 \pm 9) \boldsymbol{k J} / \boldsymbol{m o l}}{\boldsymbol{R} \boldsymbol{T}}\right) \boldsymbol{m}^{2} / \boldsymbol{s}
$$

Es bien conocido que la difusión en la fase hcp $\alpha$-Zr no cumple con la ley de Arrhenius, tanto para la auto-difusión [10] como para la difusión de elementos sustitucionales (ver [11] y las referencias citadas allí), en particular el U [7]. La desviación consiste en una curvatura con concavidad negativa, como la mostrada para la auto-difusión en la Figura 4, aunque también puede ser interpretada como un quiebre en el gráfico entre los 600 y los $650{ }^{\circ} \mathrm{C}$ (alrededor de los $900 \mathrm{~K}$ ) que marca dos regiones con distintos parámetros de difusión.

Asimismo los metales de transición $\mathrm{Fe}$, $\mathrm{Co}$ y $\mathrm{Ni}$, presentan un comportamiento denominado de difusión ultra-rápida, ya que difunden alrededor de 8 órdenes de magnitud más rápido que la auto-difusión. Su gráfico de Arrhenius también es anómalo, al igual que el resto de los difusores antes mencionados [11]. 
Experimentalmente se ve que la presencia como impureza de estos elementos, aún en cantidades de tan sólo algunos ppm, incrementa drásticamente el valor de los coeficientes de difusión, en particular el Fe, cuya presencia es inevitable en las muestras de $\mathrm{Zr}[12,13]$. Si la presencia de Fe es también la responsable del apartamiento a la ley de Arrhenius o si es consecuencia de un factor intrínseco a la fase hcp del Zr es todavía motivo de discusión en la literatura [11].

Por su parte, el Zry-4 es una aleación que en equilibrio puede considerarse como monofásica, ya que si bien contiene pequeños precipitados, estos son una proporción minúscula del volumen total del material. Estos precipitados se encuentran principalmente, en borde de grano y/o en dislocaciones [14] Debido a su ubicación y a que constituyen una proporción muy pequeña del material, sus efectos sobre el coeficiente de difusión efectivamente medido en el rango de estudio de este trabajo deberían ser despreciables. En lo que sigue trataremos de justificarlo.

La difusión en una matriz de un elemento A puede ser modificada significativamente por un aleante B. Este fenómeno ha sido verificado en distintas aleaciones $\mathrm{AB}$ binarias homogéneas. Los principales efectos del aleante son sobre las interacciones producidas entre vacante-soluto y soluto-soluto que provocan modificaciones en los tipos de saltos difusivos.

Consideremos, por ejemplo, la influencia de los átomos de soluto en una aleación muy diluida. Dependiendo de la energía de interacción, un soluto atrae o repele las vacancias y las tasas de salto de los átomos de disolvente en las proximidades del átomo de soluto se alteran. Esto influye en el coeficiente de difusión del disolvente. Cuando la concentración de soluto aumenta, el número de átomos de disolvente afectados aumenta. Dependiendo de la energía experimentada por la vacancia en las proximidades del soluto, ciertos solutos aceleran la difusión del disolvente y otros la reducen.

En aleaciones diluidas que contienen pequeñas fracciones atómicas $X_{B}$ del soluto B el coeficiente de auto-difusión del disolvente se puede escribir como:

$$
\boldsymbol{D}_{B}^{A}=\boldsymbol{D}_{B}^{A}(0) e^{b X_{B}}
$$

En aleaciones como el Zry-4 que contienen pequeñas fracciones atómicas $X_{B}<1$ del soluto B el coeficiente de difusión del disolvente se podría aproximar por:

$$
\boldsymbol{D}_{B}^{A}=\boldsymbol{D}_{B}^{A}(0)\left(1+\boldsymbol{b}_{1} \boldsymbol{X}_{B}+\ldots\right)
$$

El término $b_{1} X_{B}$ da cuenta de la modificación de los tipos de salto de los átomos de disolvente por su interacción con los átomos vecinos de soluto. Términos cuadráticos en $X_{B}$ corresponden al efecto de los pares B de átomos, etc.

En estos casos, entonces, la fracción de los elementos aleantes que se encuentran en solución serían los que podrían modificar el proceso difusivo, en tanto que la fracción que se encuentra en los precipitados no participa del fenómeno. Dada la baja solubilidad de estos aleantes en el Zr, [14] la fracción $X_{B}$ de la ec. (8) es baja y, consecuentemente, la modificación de $\boldsymbol{D}_{\boldsymbol{B}}^{\boldsymbol{A}}$ también.

La excepción a este comportamiento está dada por los aleantes ultra-rápidos, $\mathrm{Fe}$ y $\mathrm{Ni}$, ya que, como dijéramos, pequeñas cantidades de estos elementos, aún decenas de ppm, pueden modificar fuertemente los valores de $\mathrm{D}$ en matrices de $\mathrm{Zr}$, produciendo incrementos que no se rigen por la simple expresión (8). Por lo tanto, como en el caso del Zr puro, la presencia y la cantidad de estos aleantes ultra-rápidos podría ser el factor determinante a la hora de analizar los valores de $\mathrm{D}$ medidos.

Al sumar la cantidad de aleantes ultra-rápidos presentes en el Zry-4 tenemos 0,21 wt\% de Fe y 0.0032 wt $\%$ de $\mathrm{Ni}$; nos encontramos con que es una cantidad superior a la de las muestras de $\mathrm{Zr}-\alpha(0,016 \mathrm{wt} \%$ de $\mathrm{Fe})$ usadas para obtener los resultados de [7]. Si bien la relación entre incremento de D y contenido de impurezas ultra-rápidas no es lineal, consideramos que podrían explicarse los valores superiores de D de U en Zry-4 con respecto a los de D de U en $\mathrm{Zr}-\alpha$ a partir de, precisamente, este mayor contenido de Fe y Ni en Zry-4.

Además, como puede verse en las micrografías de la Figura 1, el tamaño de grano de las muestras de Zry-4 varían entre los 10 y los $20 \mu \mathrm{m}$, en tanto que la distancia media recorrida por el difundente $(4 D t)^{1 / 2}$ está entre los 0,3 y los 1,8 $\mu \mathrm{m}$, es decir entre 1 y 2 órdenes de magnitud inferior al tamaño de grano, por lo que un incremento superior al $10 \%$ en el coeficiente de difusión en volumen por efecto de la difusión por borde de grano no es esperable en este sistema. Por su parte las muestras de Zr puro usadas en [7] tenían unos pocos granos con un tamaño de varias decenas de $\mathrm{mm}$, por lo que este efecto es aún menos esperable. 
La otra diferencia entre Zr- $\alpha$ puro y Zry-4 es cualitativa; en Zry-4 la ley de Arrhenius se cumple con una entalpía de activación superior a los $300 \mathrm{~kJ} / \mathrm{mol}$, que es lo esperable para la difusión en un metal, y un $\mathrm{D}_{0}$ del orden de $10^{-2} \mathrm{~m}^{2} / \mathrm{s}$, que es un orden de magnitud superior a lo habitualmente medido en metales puros (de entre $10^{-3}$ y $10^{-6} \mathrm{~m}^{2} / \mathrm{s}$ ) aunque razonable para una aleación metálica. Por su parte en $\mathrm{Zr}-\alpha$ no es posible asignar valores a los parámetros de difusión ya que la ley de Arrhenius no se cumple.

Como dijéramos, el origen de la curvatura observada en el gráfico de Arrhenius para la mayoría de los difusores estudiados en $\alpha-Z r$ es un tema abierto. No obstante y a partir de estudios ab initio $[15,16]$ hay un cierto consenso en asignar a la presencia del Fe como impureza un papel relevante en este fenómeno, aunque no como se propusiera inicialmente en trabajos de los años 90s [17,18], referidos a la variación en la solubilidad del Fe con la temperatura en la matriz $\alpha-\mathrm{Zr}$.

En estos cálculos ab initio [15] se propuso un modelo un poco más elaborado que la simple precipitación del $\mathrm{Fe}$ como responsable del quiebre/curvatura de los difusores ultra-rápidos en $\mathrm{Zr}-\alpha$. Se mostró que la especie migrante ( $\mathrm{Fe}, \mathrm{Co}$ o $\mathrm{Ni}$ ) puede ocupar en forma estable alguno de los siguientes tres estados: (1) o bien una posición intersticial altamente móvil; (2) o bien una posición cuasi-sustitucional relativamente inmóvil, (3) o estar en una configuración tipo dumbell (2 átomos del difundente ligados, muy próximos entre sî) prácticamente inmóvil, que sirve como trampa e inhibe a estos átomos del proceso difusivo. A partir de la variación de la población de estos estados con la temperatura y de las diferentes energías asociadas a cada uno de ellos se puede ver, como en el caso de $\alpha-Z r$, el gráfico de Arrhenius se curva en el rango de temperaturas estudiado.

Este modelo fue calculado sólo para difusores ultra-rápidos y no para difusores sustitucionales como el U aunque, si bien su extrapolación a estos últimos no es directa, los resultados experimentales en [7] son congruentes con esta idea.

Por otra parte en [16] se estableció que los átomos de Fe que ocupan las posiciones intersticiales móviles tipo (1) se encuentran en una configuración cuasi-degenerada: por un lado la posición octaédrica es la más estable (es decir la de menor energía de formación) pero también existe una configuración (que fue denominada off-site) que tiene una energía de formación sólo de $0,36 \mathrm{eV}$ superior. En la configuración off-site el átomo de Fe está ligeramente desplazado desde un nodo de la red hacia la posición octaédrica, mientras que cuatro átomos vecinos del $\mathrm{Zr}$ se acercan al átomo de Fe provocando que las distancias interatómicas disminuyan a 2,5 ̊̊ (la distancia entre primeros vecinos en la red perfecta del Zr- $\alpha$ es de $3,13 \AA$ ). Esta configuración presenta distancias interatómicas similares a las de las configuraciones de los intermetálicos tales como $\mathrm{Zr}_{3} \mathrm{Fe}$ y $\mathrm{Zr}_{2} \mathrm{Fe}$ que se encuentran en el diagrama binario $\mathrm{Zr}$-Fe [19]. También en la posición octaédrica la distancia de equilibrio entre el Fe y los primeros vecinos del $\mathrm{Zr}$ es menor: 2,43 $\AA$. En ambas configuraciones, la octaédrica y la off-site, hay una fuerte interacción entre los electrones de valencia de las especies involucradas que resulta de esta disminución de las distancias interatómicas.

La presencia de Fe off-site produce una disminución, tanto en la formación de la energía de la vacancia como una distorsión de la red, con átomos de $\mathrm{Zr}$ desplazados de sus nodos en dirección a los átomos de $\mathrm{Fe}$. En [16] se calculó además que el movimiento del complejo Fe-vacancia como un todo requiere una energía de 0,4 eV más baja que el movimiento de una única vacancia.

Los precipitados presentes en el Zry-4 producto del contenido de elementos aleantes podrían estar anclando estas configuraciones intersticiales móviles, tan próximas a la estructura de los intermetálicos $\mathrm{Zr}-\mathrm{Fe}$, aún para las temperaturas más altas, próximas a $\mathrm{T}_{\alpha \beta}$ a diferencia de lo que ocurre en el $\mathrm{Zr}$ puro. Destacamos entonces que un único mecanismo sería el responsable de la difusión de U en Zry-4 en todo el rango de temperaturas estudiado lo que daría origen a un gráfico de Arrhenius recto como el observado en la figura 4, en contraposición al gráfico curvo correspondiente a la difusión de U en $\mathrm{Zr}-\alpha$ puro.

Por otra parte, encontramos una tercera diferencia entre ambas matrices al considerar la solubilidad del $\mathrm{U}$ en cada una de ellas. En tanto que todos los perfiles de difusión en $\mathrm{Zr}-\alpha$ son ajustados por la expresión gaussiana de la ec.(4), aún a las temperaturas más bajas, en el Zry-4 por debajo de $600{ }^{\circ} \mathrm{C}$ nos encontramos con perfiles de difusión como el que se muestra en la Figura 5-a: en línea llena se ve el espectro $\alpha$ para una muestra sin recocer, mientras que la línea punteada corresponde a la misma muestra luego de un recocido de 610 días (más de1 año y 8 meses) a $570{ }^{\circ} \mathrm{C}$ (para $590{ }^{\circ} \mathrm{C}$ el comportamiento es similar). A diferencia de las muestras recocidas a mayores temperaturas, el ancho del pico centrado a $4267 \mathrm{keV}$ (correspondiente al canal 362) no cambia, sino que se puede observar una cola a más bajas energías, en la zona marcada con un círculo y luego ampliada. 

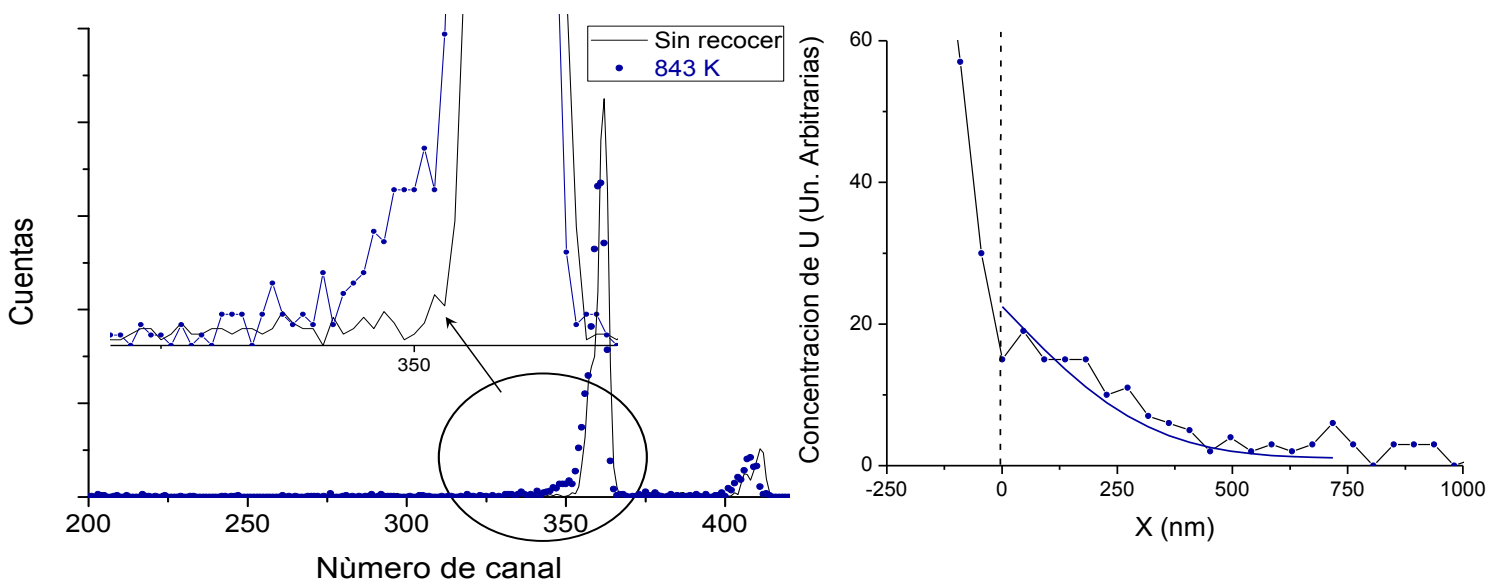

Figura 5: a-Espectros antes y después de recocer 610 días a $843 \mathrm{~K}\left(570{ }^{\circ} \mathrm{C}\right)$. b- Ajuste con función erf (ec.9)

En este caso una importante cantidad del U depositado es retenido en la superficie de la muestra aún después de haber sido recocida. Esto muy probablemente se deba a la baja solubilidad del U en el Zry-4; el punto de quiebre, en donde se inicia la cola de baja energía, corresponde al límite de solubilidad del U. De esta forma el depósito inicial funciona como una fuente constante de $\mathrm{U}$ difundente; eligiendo el origen de coordenadas $\mathrm{x}=0$ en la interfase U/Zry-4, la concentración de $\mathrm{U}$ en esa interfase es constante e igual a la solubilidad máxima del $\mathrm{U}\left(C_{0}\right.$. $)$ a $\mathrm{T}=570{ }^{\circ} \mathrm{C}$ para todo tiempo. Para esta condición de contorno la solución a la ley de Fick es:

$$
C(x)=C_{0}\left[1+e r f\left(\frac{x}{\sqrt{4 D t}}\right)\right]
$$

Aplicando la ec.(3) y transformando la coordenada $\mathrm{N}^{\mathrm{ro}}$. de Canal en profundidad (medida en $\mathrm{nm}$ ), obtenemos la línea con puntos de la Figura 5-b, que al ser ajustada con la función de la ec.(9) (línea llena) nos da un valor de $\mathrm{D}=6,1 \times 10^{-22} \mathrm{~m}^{2} / \mathrm{s}$, valor que se alinea perfectamente bien con los coeficientes de difusión medidos a temperaturas superiores, donde la mayor solubilidad del U nos permitió ajustar con una Campana de Gauss como la de la ec.(4). De esta forma podemos concluir que las hipótesis usadas (baja solubilidad del $\mathrm{U}$ y ajuste con una función error) son adecuadas, tanto para 570 como para $590{ }^{\circ} \mathrm{C}$, donde se obtiene un espectro similar luego de un recocido de 124 días.

Finalmente remarcaremos que estas mediciones a bajas temperaturas y bajas solubilidades, donde la penetración promedio del difundente (4Dt) ${ }^{1 / 2}$ es de alrededor de $300 \mathrm{~nm}$ nos permiten testear el límite inferior de detección de la espectrometría $\alpha$ con resultados satisfactorios. El límite de error cercano al $30 \%$ para estas dos temperaturas más bajas, es producto de agregar un factor adicional al cálculo de error respecto a las mediciones hechas a mayores temperaturas. El error en la determinación de $D$ [1] habitualmente proviene de sumar el error estadístico con el que se determina la pendiente del la recta de ajuste al linealizar la ec.(4) (o la 9) con la propagación del error con el que se determina la coordenada $\mathrm{x}$ a partir del poder de frenado de las partículas $\alpha$ en la materia. Este error suele ser de alrededor de un $10 \%$ del valor medido.

Habitualmente al seleccionar el número de puntos del espectro que se utilizaran para realizar el ajuste, la adición o substracción de uno o dos puntos no provoca una variación significativa en el valor final del ajuste. A estas bajas temperaturas, como la penetración es menor, el número de puntos del espectro usados para el cálculo es también menor, por lo que al agregar o quitar algún punto esta variación puede (y en nuestro caso, lo es) ser percibida, introduciendo un factor extra al error que al ser tenido en cuenta nos lleva a un total cercano al $30 \%$ tal como fue reportado.

\section{CONCLUSIONES}

Se midieron los valores de los coeficientes de difusión del U en Zry-4 entre 570 y $820^{\circ} \mathrm{C}$ ( 843 y $1093 \mathrm{~K}$ ) usando la espectrometría $\alpha$ y se compararon estos valores con los de la difusión de $\mathrm{U}$ en $\mathrm{Zr}$ - $\alpha$ puro medidos en un rango de temperaturas similar, encontrándose 3 diferencias significativas:

(1) Los valores de D, a temperaturas mayores a los $600{ }^{\circ} \mathrm{C}$, son sistemáticamente mayores en Zry-4 
con respecto al Zr- $\alpha$; esto puede deberse tanto a la mayor presencia de impurezas ultra-rápidas en la primera matriz, como, en menor medida, a la diferencia de microestructura entre ambas matrices.

(2) La dependencia de D con la temperatura obedece a la ley de Arrhenius en el caso del Zry-4, en tanto que para Zr- $\alpha$ se observa una curvatura con concavidad negativa en el gráfico de Arrhenius. Se postula como posible razón para esta diferencia que uno o varios de los mecanismos de difusión en $\mathrm{Zr}-\alpha$, que involucran la movilidad de complejos Fe-Vacancia, podrían quedar anclados en los pequeños precipitados presentes en el Zry-4 producto de la interacción de los distintos aleantes con el Zr.

(3) La solubilidad del U en Zry-4 es menor a la solubilidad en Zr- $\alpha$, por lo menos a temperaturas inferiores a los $600{ }^{\circ} \mathrm{C}$. Nuevamente la responsable de dicha diferencia sería la presencia de los aleantes, que podrían estar compitiendo con el U para ubicarse en posiciones sustitucionales de la red.

\section{BIBLIOGRAFÍA}

[1] PÉREZ, R.A., GORDILLO J.A. Y., DI LALLA N. "Application of $\alpha$-spectrometry to the study of U diffusion in nuclear reactor materials", Measurements v.45, n.7, pp. 1836-1841. 2012.

[2] MEHRER H. (editor), Landolft-Börnstein: Diffusion in solid metals and alloys. 1 ed. Berlin Heidelberg Spenger-Verlag. 1990.

[3] PÉREZ, R.A. GORDILLO, J.A.Y., DI LALLA, N. "Experimental determination of U diffusion in $\alpha$-Ti", Philosphical Magazine A v.93, n.17, pp. 2219-2227, 2013.

[4] KÖPPERS M., HERZIG CHR., FRIESEL M., et al., Intrinsic Self-Diffusion and sustitucional Al diffusion in $\alpha$-Ti, Acta Materialia v.45 n.10, pp.4181-4191, 1997.

[5] FEDOROV G.B., SMIRNOV E.A. Diffusion in Reactor Materials 1 ed. Switzerland Trans Tech Publications, 1984.

[6] FEDOROV G.B., SMIRNOV E.A., ZHOMOV F.I., et al., Metall Metalloved Chistych Metal, v.9 pp. 30$35,1971$.

[7] GORDILLO J.A, PÉREZ R.A., DI LALLA N.,et al., "Experimental determination of U diffusion in $\alpha$ Zr", Journal of Nuclear Material, v.462, pp.85-90. 2015.

[8] CHU C W.K., Rutherford backscattering spectrometry - ASM Handbook. v. 10, Materials Characterization, Materials Park - USA, ASM International. 1986.

[9] ZIEGLER, J.F., BIERSACK, J.P., ZIEGLER M.D., SRIM - The Stopping and Range of Ions in Matter, 1 ed. Maryland, Ed. Chester, SRIM Co. 2008.

[10] HORVÁTH J., DYMENT F., MEHRER H. "Anomalous self-diffusion in a single crystal of $\alpha$ zirconium”, Journal of Nuclear Materials v.126 pp.206-214, 1984.

[11] PÉREZ R.A., NAKAJIMA H., DYMENT F., "Diffusion in $\alpha$-Ti and $\alpha$-Zr", Material Transactions, v.44 pp.2-13. 2003.

[12] LÜBBEHUSEN, M., VIEREGGE, K., HOOD, G.M., et al., "Self-diffusion in $\alpha$-Zr single crystals", Journal of Nuclear Materials v.182, pp.164-169, 1991.

[13] PÉREZ, R.A, DYMENT, F., "Diffusion of Gold in alpha-Zr", Diffusion in solids and liquids, v.2 pp.539-544, Aveiro, 2005.

[14] G. P. SABOL Y E. R. BRADLEY (editors), Zirconium in the Nuclear Industry: Eleventh International Symposium. 1 ed.,West Conshohocken, ASTM International, 1996.

[15] PASIANOT R., PÉREZ R.A., "First-principles appraisal of solute ultra-fast diffusion in hcp Zr and Ti", Journal of Nuclear Material v.434 n.1-3, pp.158-161, 2013.

[16] PASIANOT R., PÉREZ R.A., "Issues in the ab-initio assessment of self diffusion: the cases of Zr and Ti”, Physica B v407 n.16, pp.3298-3300, 2012.

[17] HOOD G. "The anomalous self-diffusion in $\alpha$-Zr", Journal of Nuclear Material, v.135, pp.292-295, 1985.

[18]FRANK W. "Rapid pair enhancement diffusion in $\alpha-\mathrm{Zr}$ and its integration into the point defect scenario in hep metals", Philosophical Magazine A , v.63, pp.897-913, 1991.

[19] ARIAS D., ABRIATAJ, P. "Fe-Zr (Iron-Zirconium). Binary Alloy Phase Diagram", In: $2^{\text {nd }}$ ed. ASM International USA, pp.1798-1800, 1990. 